\title{
Comment Vidéo Digest s'intégrera-t-il dans la loi HPST ?
}

\section{Heresbach}

Alors que Vidéo Digest 2009 célèbre ses 20 ans dont le succès repose sur l'association de sessions en direct et de sessions plus académiques et thématisées, il n'échappe à personne que ce succès ne se pérennisera que s'il s'inscrit dans le DPC ou développement professionnel continu. En effet, la crise dite financière, même si certains prédisent sa fin proche, aura été également l'occasion pour nos partenaires d'ajuster ou de réviser leur politique qui a peu de chance de bouger, même lors de la « reprise». La loi HPST définit le DPC qui comporte six objectifs dont l'EPP et le perfectionnement des connaissances. Il est donc logique pour ne pas dire certain que la participation à Vidéo Digest s'intégrera dans le deuxième objectif. La méfiance réside plutôt dans les modalités et le profil d'organisation du DPC qui, outre un organisme gestionnaire incluant une commission scientifique indépendante (CSI), comporte aussi un conseil national du DPC (CNDPC) et le Conseil de l'ordre. Alors que le CSI aura pour mission d'évaluer les organismes de formation, c'est le CNDPC, placé auprès du ministre et composé de personnalités choisies, qui définira les modalités d'organisation, les critères d'enregistrement et les priorités du DPC. Si les objectifs de FMC et d'EPP ne seront probablement pas modifiés par rapport à l'existant, les objectifs de la gestion des risques, ceux de santé publique et de maîtrise des dépenses de santé présentent plus d'incertitude, même si l'on peut prédire que ceux-ci seront pilotés ou sous la gouverne des ARS. Ainsi, les organismes accrédités pour la FMC garderont probablement leur accréditation et ceux mandatés pour l'EPP devront s'adapter et/ou se regrouper. Cette « nouvelle » organisation verra-t-elle le jour ou encore une fois s'agirat-il d'un faux départ? La nécessité de disposer de décret en conseil d'État pour l'application de la loi HPST pourrait laisser penser à une certaine nonchalance, mais l'intégration dans cette loi de la maîtrise des dépenses de santé et la définition des objectifs de santé publique laissent penser que cette nouvelle donne sera la bonne. Une fois de plus, les composantes de la spécialité, en particulier impliquées dans l'endoscopie digestive, devront être vigilantes pour une reconnaissance de l'endoscopie à son juste niveau, aussi bien dans les choix de maîtrise négociée des coûts de l'endoscopie que dans l'organisation de l'endoscopie diagnostique ou thérapeutique et de la qualité ou sécurité de l'endoscopie. Alors, plus que jamais, la SFED en tant que société savante doit rester impliquée en première ligne dans les composantes FMC, qualité-sécurité et coopérer avec les autres acteurs (syndicats, URML, EPPHGE) pour les autres objectifs définis par l'article 59 de la loi HPST. Ceci, comme suggéré dans le précédent éditorial, invite la SFED à développer une rubrique juridique dans Acta Endoscopica. Vous comprendrez que même si ces publications n'ont pas de valeur opposable et n'auront que valeur d'avis d'expert, elles nécessitent plus d'une lecture pour arriver à un consensus non seulement médical mais aussi juridique conforme. Cette rubrique débute dès ce numéro et se terminera par une fiche SFED sous la forme de questions-réponses pratiques : «Le juridique et l'endoscopie en pratique » Toute les questions sur ce sujet sont les bienvenues afin de mieux répondre à vos préoccupations. 